\title{
Tratamiento endovascular de la coartación aórtica nativa $y$ recurrente en pacientes mayores de 4 años: Resultados y complicaciones
}

Martín Valdebenito, Alejandro Martínez, Gonzalo Martínez, Nicolás Veas, Dante Lindefjeld, Francisco Garay, Felipe Heusser, Daniel Springmüller, Alejandro Fajuri, Alejandra Flores, Francisco Vergara.

Departamento de Enfermedades Cardiovasculares, Pontificia Universidad Católica de Chile.

Objetivos: Reportar nuestra experiencia en el tratamiento endovascular de la Coartación Aórtica (CoAo). Métodos: Se revisaron los registros clínicos de los pacientes mayores de 4 años intervenidos por una CoAo nativa o recoartación en el Hospital Clínico de la Universidad Católica entre los años 2007 y 2012. Se realizaron estadísticas descriptivas y se utilizó el test de Wilcoxon signed rank, con una $\mathrm{p}<0,05$.

Resultados: En ese período se realizaron 27 intervenciones en pacientes con CoAo. El promedio de edad fue 20.6 años ( 5 - 64); 8 (30\%) eran mujeres y 8 (30\%) menores de 14 años. Quince pacientes (55\%) tenían una cardiopatía congénita asociada, de los cuales 9 (33\%) tenían válvula aórtica bicúspide, 3 (11\%) una comunicación interventricular (CIV) y 3 co-existencia de ambas entidades (11\%). Nueve (33\%) pacientes eran hipertensos. En 20 (74\%) pacientes la intervención fue sobre una coartación aórtica nativa y en 7 (26\%) sobre una recoartación ( 2 con cirugía previa y 5 con angioplastia anterior). Se implantó un stent en 23 (85\%) pacientes y se utilizó sólo balón en 4 (15\%). En 26 pacientes (96\%) se logró una reducción del gradiente a menos de $20 \mathrm{mmHg}$ (gradiente pre $32 \mathrm{vs}$ gradiente post $6 \mathrm{mmHg}$, $\mathrm{p}<0,01)$. Hubo complicaciones del sitio de punción en 3 pacientes ( 2 hematomas y 1 disección focal en el origen de la arteria ilíaca externa) y complicaciones mayores en 2 pacientes (disección aórtica tipo B) que requirieron tratamiento intervencional (uno percutáneo y uno quirúrgico). No hubo mortalidad en nuestra serie.

Conclusiones: La intervención endovascular es una opción de tratamiento efectiva para la CoAo.

Keywords: Coartación de la aorta, reparación endovascular.

Correspondencia:

Dr. Alejandro Martínez.

Departamento de Enfermedades Cardiovasculares,

Pontificia Universidad Católica de Chile,

amartine@med.puc.cl 


\section{Endovascular Repair of Native and Recurrent Aortic Coarctation in patients older than 4 years of age}

Aim: There is an increasing interest in the endovascular therapy of both native and recurrent aortic coarctation (Aco). In this article we report the results of endovascular treatment of ACo in children and adults at our institution, from 2007 to 2012.

Method and Results: The clinical, angiographic and hemodynamic data of all patients submitted to endovascular repair of ACo at the Endovascular Therapy Center of the Catholic University Hospital were reviewed. Children under 4 years of age were excluded. A total of 27 subjects were included. Mean age was 20.6 years (range 5-64); 30\% were females and $30 \%$ were under 14 years of age. 15 patients had associated congenital defects: bicuspid aortic valve (9), VSD (3), or both VSD and bicuspid aortic valve (3). Hypertension was present in $33 \%$ of patients. $74 \%$ had endovascular repair of a native Aco and $26 \%$ of a re-coarctation ( 2 of them post surgical treatment and 5 post endovascular repair). $85 \%$ of cases had stent implantation. Repair resulted in a significant $(\mathrm{p}<0.01)$ decrease in aortic pressure gradient (mean 32 vs $6 \mathrm{mmHg}$ ). There was no mortality associated to the procedure. Two patients developed aortic dissection post procedure, successfully treated by surgery or endovascular repair. There were no instances of stent migration.

Conclusion: Endovascular repair is an effective means of correcting Aco. Potential complications need careful technique and monitoring during the procedure. Keywords: Aortic coarctation, endovascular repair.

\section{Introducción}

La Coartación Aórtica determina un aumento de la poscarga del ventrículo izquierdo, hipertensión proximal y disminución de la perfusión en la parte inferior del cuerpo. Cuando no es corregida, su evolución natural se asocia a disminución de la sobrevida, fundamentalmente por complicaciones asociadas a la hipertensión arterial. La opción de tratamiento inicial fue la resección quirúrgica con anastomosis termino-terminal, descrito por primera vez por Crafoord en $1945^{1}$. Sin embargo, la cirugía conlleva riesgos mayores, por lo que en los últimos años su tratamiento endovascular ha ganado aceptación y actualmente es preferido en niños mayores de 4 años y adultos. 2,3,4

En este artículo reportamos la experiencia en nuestro centro con el tratamiento endovascular de la coartación aórtica nativa y recurrente. El objetivo de nuestro estudio fue evaluar la eficacia del procedimiento, determinar sus complicaciones agudas y los factores de riesgo para su incidencia.

\section{Métodos}

\section{Pacientes}

Se analizaron los registros de todos los pacientes intervenidos en forma endovascular por coartación aórtica con angioplastía, con o sin stent, en el Hospital Clínico de la Universidad Católica, entre los años 2007 y 2012.
La decisión de tratar de esta forma o con cirugía fue dependiente del tratante, sin un criterio definido para su elección.

Se incluyeron pacientes con Coartación aórtica nativa o recurrente alejada y localizada en el istmo. Se excluyeron pacientes menores de 4 años de edad.

Se analizó la información demográfica de los pacientes, los diagnósticos asociados, las características de la coartación aórtica y del procedimiento realizado, además de las complicaciones agudas encontradas.

\section{Definiciones}

Coartación Aórtica: El estrechamiento del lumen aórtico, habitualmente inmediatamente distal a la subclavia izquierda, se consideró significativo cuando, además de la confirmación anatómica (por tomografía computada (TAC), Resonancia Magnética o ecocardiografía), se asoció a una diferencia de presión arterial sistólica (PAS) entre miembros superiores e inferiores $>20 \mathrm{mmHg}$ o un diferencial de PAS $<20 \mathrm{mmHg}$ en presencia de Hipertensión Arterial o Hipertrofia ventricular Izquierda (HVI).

Resultado Exitoso: Gradiente sistólico residual < $20 \mathrm{mmHg}$ posterior a la angioplastía, en ausencia de complicaciones mayores (muerte, requerimientos de cirugía, discapacidad permanente).

Complicaciones agudas: Complicaciones derivadas del 
procedimiento que requirieron procedimientos o terapias adicionales durante la intervención, previo o posterior al alta hospitalaria. Estas fueron categorizadas como complicaciones de la pared aórtica, vasculares periféricas y técnicas.

Complicaciones de la pared aórtica: Incluyeron desgarros intimales, disección (incluyendo ruptura de la pared aórtica), o el desarrollo de un aneurisma.

Aneurisma: fue definido como una expansión de la pared aórtica $>10 \%$ del lumen nativo adyacente que no estaba presente previo a la intervención. Disección fue definida como una extravasación de contraste fuera del lumen del vaso, y desgarro intimal como un defecto de llenado observado dentro del lumen del vaso sin evidencia de extravasación fuera del lumen (proximal o distal al stent previamente instalado).

Complicaciones vasculares periféricas: Incluyeron accidentes vasculares encefálicos (AVE), embolias periféricas, o lesiones vasculares significativas que requirieron reparación quirúrgica o prolongación de la hospitalización.

Complicaciones técnicas: Incluyeron ruptura de balón o migración del stent fuera del balón, durante o después de la liberación.

\section{Análisis Estadístico}

Se usaron estadísticas descriptivas, tales como media, desviación estándar y frecuencia, las que fueron calculadas para cada característica clínica y demográfica si era apropiada. Se utilizó el "signed rank test" de Wilcoxon para evaluar diferencias de variables con distribución no paramétrico. Se estableció $\mathrm{p}<0.05$ como criterio de significación.

\section{Resultados}

\section{Características de los pacientes}

Todos los procedimientos se realizaron en el Centro

\begin{tabular}{|l|l|}
\hline \multicolumn{2}{|c|}{ Tabla 1 Características de los Pacientes. } \\
\hline Sexo & \\
\hline Femenino & $8(30 \%)$ \\
Distribución por Edad & \\
$4-9$ años & $6(22 \%)$ \\
$10-19$ años & $12(45 \%)$ \\
$20-29$ años & $3(11 \%)$ \\
30-39 años & $3(11 \%)$ \\
$>40$ años & $3(11 \%)$ \\
Promedio de Edad & $20.6(5-64)$ \\
Hipertensión Arterial & $9(33 \%)$ \\
Cardiopatía Congénita Asociada & $15(55 \%)$ \\
Válvula Aórtica Bicúspide (VAB) & $9(33 \%)$ \\
Comunicación Interventricular (CIV) & $3(11 \%)$ \\
VAB + CIV & $3(11 \%)$ \\
Síndrome de Turner & $1(3.7 \%)$ \\
\end{tabular}

de Terapia Endovascular del Hospital Clínico de la Universidad Católica. La primera intervención registrada en nuestro estudio se efectuó el 13 de Marzo del 2007 y la más reciente el 30 de Julio del 2012. Fueron realizadas 27 angioplastías para el tratamiento de la coartación aórtica nativa o recurrente dentro de ese período. Las característica de los pacientes se señalan en la Tabla 1.

\section{Características de la Coartación}

La Coartación Aórtica Nativa estuvo presente 20 pacientes (74\%). De los 7 pacientes con coartación aórtica recurrente, 5 tuvieron angioplastías previas y los otros 2 habían sido operados (Tabla 2).

Tabla 2. Características de la Coartación

\section{Tipo de Coartación \\ Nativa \\ Recurrente \\ Angioplastía previa \\ Cirugías previas}

\section{Procedimiento}

En todos los casos la intervención se realizó por vía femoral y una vez retrogradada la coartación se instaló una guía semi-rígida en la arteria subclavia derecha o izquierda. En el 85\% de los casos se implantó un stent. En estos casos, previo al implante se colocó un introductor largo a través de la coartación, para llevar el stent al sitio estenótico. En niños pequeños (menos de $35 \mathrm{Kg}$ ) o en los casos que tenían un stent previo, se realizó angioplastía sólo con balón. El diámetro de los dispositivos no superó el de la aorta proximal a la coartación. Para guiar la dilatación, en los primeros casos se obtuvo aortografía contrastada en múltiples proyecciones, pero desde el año 2010 se utiliza aortografía rotacional con reconstrucción y "roadmaping" 3D, lo que permite ahorrar tiempo de radioscopía y medio de contraste (Figura 1).

Figura 1: Uso de herramienta 3D roadmaping para el posicionamiento de stent en sitio de coartación aórtica.

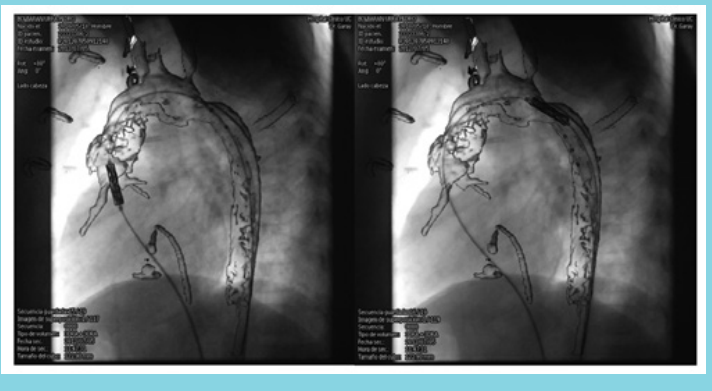


El cierre del acceso femoral se hizo con compresión manual o con dispositivo Prostar ${ }^{\circledR}$. La estadía hospitalaria fue en promedio de 2,4 días (1-5 días), presentando una mayor estadía hospitalaria los pacientes que presentaron complicaciones. (Tabla 3).

\begin{tabular}{|l|l|}
\multicolumn{2}{c}{ Tabla 3 } \\
\hline Angioplastía con Stent & $23(85 \%)$ \\
Angioplastía sin Stent & $4(15 \%) *$ \\
Número de Stent (23) & $1(93 \%)$ \\
1 & $2(7 \%)$ \\
2 & $14(59 \%)$ \\
Tipo de Stent & $8(33 \%)$ \\
CP (recubierto) & $2(8 \%)$ \\
V12 (recubierto) & 2.4 días (1-5 días) \\
Palmaz (no recubierto) & \\
Estadía Hospitalaria &
\end{tabular}

$*=1$ por bajo peso $<35 \mathrm{Kg} .3$ por tener angioplastias con stent previas

\section{Éxito del Procedimiento}

Se logró un resultado exitoso en 25 de los 27 pacientes $(93 \%)$. En ellos, la gradiente sistólica entre la aorta ascendente y descendente bajó desde una media de 32 a $6 \mathrm{~mm} \mathrm{Hg}(\mathrm{p}<0.01)$ y no se presentaron complicaciones mayores. Figura 3.

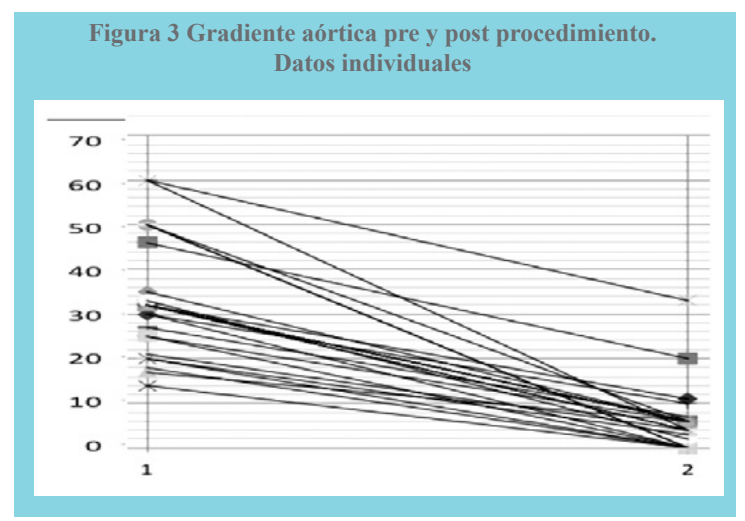

Dos procedimientos fueron considerados no exitosos. Ambos requirieron de una cirugía adicional por complicaciones y en uno de ellos, además, no se llegó a un gradiente $<20 \mathrm{mmHg}$ ).

\section{Complicaciones}

Se presentaron complicaciones en 5 de los 27 pacientes (18\%). Sólo en 2 casos (7.4\%), hicieron calificar al procedimiento como no exitoso y correspondieron a complicaciones de la pared aórtica.

a) Complicaciones de la Pared Aórtica: En 2 pacientes $(7,4 \%)$ hubo complicaciones de la pared aórtica.

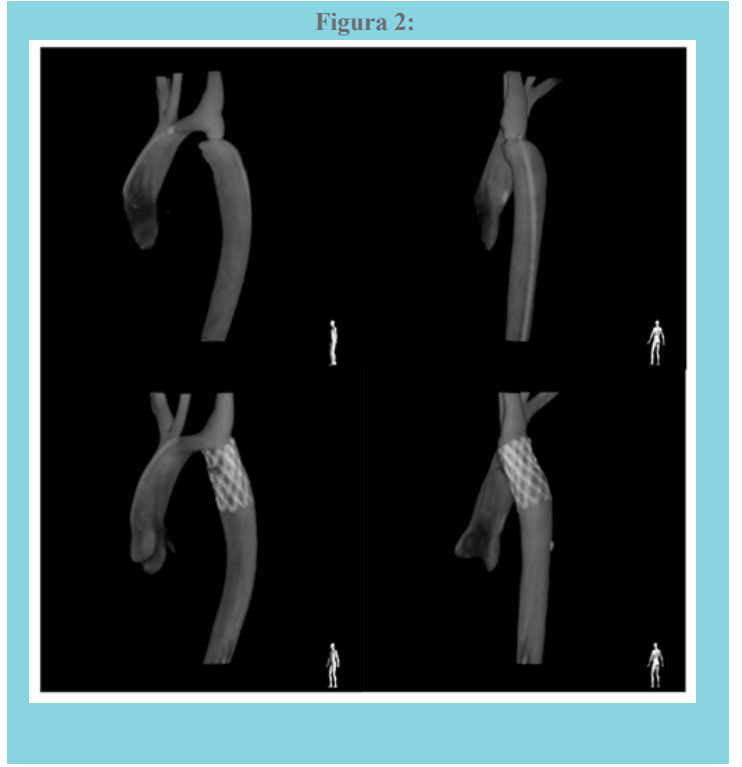

Imagen obtenida mediante una reconstrucción $3 D$ de una aortografia rotacional en sala de hemodinamia. Muestra un paciente adulto con una coartación aórtica nativa a nivel del istmo con algún grado de hipoplasia del arco transverso, en sus proyecciones oblicua anterior izquierda y posterior, en su control basal y post angioplastía.

Ambos tuvieron una disección aórtica tipo B. Los dos se diagnosticaron después del alta hospitalaria. Uno se presentó con dolor torácico al día siguiente de su alta, comprobándose una disección que se extendía desde el extremo distal del stent hasta la aorta abdominal, y se trató con el implante de dos endoprótesis por vía endovascular. El segundo se manifestó como claudicación intermitente, semanas después de la intervención, cuando se demostró una disección desde el origen de las arterias renales hacia distal. Este paciente se trató con un bypass aortobifemoral, mediante cirugía abierta. Ambas se presentaron en pacientes de mayor edad (44 y 45 años) y coincidieron en el uso de un stent no recubierto (Palmaz).

b) Complicaciones Vasculares Periféricas: Se presentaron en 3/27 pacientes (11\%). Dos correspondieron a hematoma del sitio de punción y una a disección focal en el origen de la arteria ilíaca externa, sin consecuencias.

No tuvimos complicaciones técnicas como migración del stent o ruptura de balón.

\section{Discusión}

Esta experiencia confirma una alta tasa de éxito en el tratamiento percutáneo de la Coartación Aórtica. Se logró un resultado exitoso en un $93 \%$ de los casos, lo que es similar a lo reportado en series internacionales $\mathbf{2 , 5 , 6}$. Sin embargo, este procedimiento no está libre de riesgos. 
En esta serie, 2 pacientes $(7 \%)$, presentaron como complicación una disección aórtica. Aunque ellos fueron finalmente tratados satisfactoriamente, por ser ésta una complicación potencialmente fatal, nos plantea la necesidad de prevenir su incidencia y de establecer métodos seguros para su diagnóstico.

La disección aórtica ha sido asociada con la angioplastia de la coartación, tanto con balón como con stent $\mathbf{3 , 7}$. Puede ser debida a la disrupción de la íntima por el traumatismo de la pared aórtica en el sitio de dilatación, pero también puede deberse a lesiones provocadas por el avance de guías y catéteres. De hecho, en nuestra experiencia, en un paciente la disección se produjo desde el sitio de dilatación, en cambio en el otro se disecó la aorta abdominal, lejos de la coartación. Este segundo caso seguramente podría haber sido prevenido con mayor pulcritud técnica. El otro, en cambio, se debió directamente al traumatismo de la zona coartada, a pesar de haber sido prudentes en el diámetro del balón utilizado. Este caso, representa el 3,7\% de nuestra población total, pero ocurrió en uno de los tres pacientes mayores de 40 años.

Al igual que en otras series y en reportes de casos, la mayor edad parece tener un rol importante en complicaciones de este tipo $^{\mathbf{8 , 9 , 1 0 , 1 1}}$. Puede favorecerse por una distensibilidad disminuida de la pared aórtica que se produce solo con la mayor edad ${ }^{12,13}$, pero además por los cambios de la pared aórtica producto de la coartación. Probablemente, por los trastornos del flujo secundarios, la coartación conduce en el tiempo a cambios degenerativos de la pared, como adelgazamiento, necrosis media quística y calcificación ${ }^{\mathbf{1 4}}$. Estas condiciones harían que la aorta del adulto sea menos "tolerante" a la expansión comparado a una aorta de un niño o adolescente. Existe un reporte ${ }^{6}$ que plantea una evaluación previa con eco intravascular en pacientes de alto riesgo, con la esperanza de identificar segmentos enfermos de la aorta (necrosis media quística, nódulos calcificados) y cambiar la aproximación del procedimiento. Por otra parte, aunque no se ha comprobado, se sospecha que el uso de stent cubierto puede disminuir esta complicación; de hecho, en nuestros 2 casos en que se presentó usamos stent no cubierto. Por lo tanto, basados en nuestra experiencia, recomendamos usar stent cubierto y ser más cautos con la dilatación en los pacientes mayores. A esta edad puede ser más prudente el objetivo hemodinámico que la resolución angiográfica de la estenosis.

En nuestra experiencia fue llamativo que en los 2 casos la disección de la aorta se diagnosticó después del alta. Esta alteración no se observó en la angiografía de control posterior al implante. Probablemente la calidad de las imágenes obtenidas no fue suficiente para verla. Por lo anterior, en la actualidad recomendamos realizar angiografía con reconstrucción 3D o angio-TAC, para evaluar el procedimiento.

Desde el punto de vista de otras complicaciones, afortunadamente no tuvimos casos de AVE ni de embolias periféricas. Seregistraron lesiones vasculares significativas en 1 paciente con una disección focal en el origen de la arteria ilíaca externa y en otros 2 pacientes hematomas del sitio de punción que requirieron compresión y observación, sin necesidad de transfusiones. Tampoco tuvimos complicaciones técnicas en los procedimientos. En este sentido, una de las complicaciones que se han descrito es la migración del stent, con una incidencia entre $0 \%$ a $6.1 \%$ en otros reportes. ${ }^{2,5,7}$.La segunda complicación técnica descrita es la ruptura del balón durante la liberación del stent. Reportes describen esta complicación entre 0-4\% de los casos. ${ }^{2,5,7}$.

Por último, es importante recalcar que en esta publicación sólo reportamos los resultados y complicaciones precoces del procedimiento. No hemos completado el seguimiento, pero sabemos, por otras series, que tal como ocurre con la intervención quirúrgica, la reparación endovascular se asocia a complicaciones alejadas, como recoartación y formación de aneurisma. La incidencia de estas complicaciones, aunque no se conoce con exactitud, fluctúa entre el 1 y el 15\%, dependiendo de la técnica utilizada. Aparentemente, su aparición disminuye significativamente con el uso de stents, particularmente con stents cubiertos ${ }^{\mathbf{1 5}}$, pero aun así, estos pacientes deben ser seguidos con imágenes.

En suma, nuestra experiencia confirma que el tratamiento endovascular de la coartación aórtica, aunque potencialmente de riesgo, tomando las precauciones en la técnica y evaluación comentadas, debería ser elegible en la mayoría de los pacientes con esta patología. 


\section{Referencias:}

1 CRAFOORD C, NYLIN G. Congenital coarctation of the aorta and its surgical management. J Thorac Cardiovasc Surg 1945; 14:347-361.

2 HAMDAN MA, MAHESHWARI S, FAHLEY JT, HELLENBRAND WE. Endovascular stents for coarctation of the aorta: Initial results and intermediate-term follow up. J Am Coll Cardiol 2001; 38:1518-1523.

3 JOHNSTON TA, GRIFKA RG, JONES TK. Endovascular stents for the treatment of coarctation of the aorta: Acute results and follow-up experience. Catheter Cardiovasc Interv 2004;62:499-505.

4 SHAH L, HIJAZIZ, SANDHU S, JOSEPHA, CAOQL. Use of endovas- cular stents fro the treatment of coarctation of the aorta in children and adults: Intermediate and midterm results. J Invas Cardiol 2005;11:614-618.

5 HARRISON DA, MCLAUGHLIN PR,LAZZAM C, CONNELLY M, BENSON LN. Endovascular stents in the management of coarctation of the aorta in the adolescent and adult: One year follow up. Heart 2001;85:561-566.

6 PEDRA CA, FONTES VF, ESTEVES CA, ARRIETA SR, BRAGA SL, JUSTINO $\mathrm{H}$, et al. Use of covered stents in the man- agement of coarctation of the aorta. Pediatr Cardiol 2005;26: 431-439.

7 SUAREZ DE LEZO J, PAN M, ROMERO M, MEDINA A, SEGURA J, LAFUENTE M, et al. Immediate and follow-up findings after stent treatment for severe coarctation of aorta. Am J
Cardiol 1999;83:400-406

8 KORKOLASJ,TCHERVENKOV CI,SHUM-TIM D, ROY N Aortic rup- ture after stenting of a native coarctation in an adult. Ann Thorac Surg 2002;74:936.

9 VARMA C, BENSON LN, BUTANY J, MCLAUGHLIN PR. Aortic dissection after stent dilation for coarctation of the aorta: a case report and literature review. Catheter Cardiovasc Interv 2004;59:528-535.

10 CHESSA M, CARROZZA M, BUTERA G, PIAZZA L, NEGURA DG, BUSSADORI C, et al. Results and mid-long- term follow-up of stent implantation for native and recurrent coarctation fo the aorta. Eur Heart J 2005;26:2728-2732.

11 FEJICZ, VAN OORT A. Fatal dissection of the descending aorta after implantation of a stent in a 19 year old female with Turner syndrome. Cardiol Young 2005;15:529-531.

12 KUECHERER HF, JUST A, KIRCHHEIM HR. Evaluation of aortic compliance in humans. Am J Physiol Heart Circ Physiol 2000;278: H1411-H1413.

13 LAKATTA E. Age-associated cardiovascular changes in health:Impact on cardiovascular disease in older persons. Heart Failure Rev 2002;7:29-49.

14 ISNER JM, DONALDSON RF, FULTON D, BHAN I, PAYNE DD, CLEVELAND RJ. Cystic medial necrosis in coarctation of the aorta: a potential factor contributing to adverse consequences observed after percutaneous balloon angioplasty of coarctation sites. Circulation 1987;75:689-695.

15 BRUCKHEIMER E, CARDOSO CA. Stenting options for coarctation of the aorta. Intervent Cardiol Clin 2013;2:115-129 